\title{
Homoclinic Orbits and Chaos in a Second-Harmonic Generating Optical Cavity
}

\author{
Alejandro Aceves ${ }^{\mathrm{a}}$, Darryl D. Holm ${ }^{\mathrm{b}}$, Gregor Kovačič ${ }^{\mathrm{c}}$, \\ Ilya Timofeyev ${ }^{\mathrm{c}}$ \\ a Department of Mathematics and Statistics, University of New Mexico, \\ Albuquerque, NM 87131 \\ b Theoretical Division, Los Alamos National Laboratory, Los Alamos, NM 87545 \\ c Mathematical Sciences Department, Rensselaer Polytechnic Institute, Troy, NY \\ 12180
}

\begin{abstract}
We present two large families of Šilnikov-type homoclinic orbits in a two modemodel that describes second-harmonic generation in a passive optical cavity. These families of homoclinic orbits give rise to chaotic dynamics in the model.
\end{abstract}

\section{Introduction}

A standard model of the dynamics of light in a second-harmonic generating passive optical cavity consists of the equations

$$
\dot{A}_{1}=-i \kappa_{1} A_{1}+i A_{2} A_{1}^{*}-\varepsilon \alpha A_{1}, \quad \dot{A}_{2}=\frac{i}{2} A_{1}^{2}-\varepsilon\left(i \kappa_{2}+\alpha\right) A_{2}+\varepsilon \gamma_{2},
$$

where $A_{1}$ and $A_{2}$ are the slowly-modulated amplitudes of the fundamental color and its second harmonic, $\kappa_{1}$ and $\varepsilon \kappa_{2}$ are the frequency mismatches, $\varepsilon \alpha A_{1}$ and $\varepsilon \alpha A_{2}$ are the losses of light in the ring cavity, and $\varepsilon \gamma_{2}$ is the external pumping of the second-harmonic mode [1-4]. In order to derive this model, one must assume that the crystal in the cavity does not have a cubic symmetry. Crystals without this symmetry are, for instance, KTP or $\mathrm{LiNbO}_{3}$. Moreover, the crystal must be short, so that all the spatial effects in it, such as the modulational instability, can safely be neglected [5]. One must also assume that no modes other than a fundamental frequency of the light and its second harmonic can be present in the cavity $[1,4]$. Finally, one must assume that the cavity is of extremely high quality, so that both the pumping and the losses can be taken as relatively small and considered as perturbation terms. 
The model (1) and its various modifications display a surprisingly rich array of different types of dynamical behavior. These include bistability, stable periodic pulsations, and chaos [1-4], which were observed both numerically and also experimentally. Thus, it seems appropriate to investigate mathematical properties of the model (1) that could shed light on these types of behavior, and the chaotic dynamics in particular, which we do in this letter.

We use the results of [6-8] to compute two large families of homoclinic orbits that the model (1) supports. All these orbits are of Šilnikov type [9-13], and bring along with them chaotic dynamics arising from a Smale horseshoe construction [14]. Physically, these dynamics should manifest themselves by transient intermittent flickering of light between the fundamental and the second-harmonic colors.

\section{The ideal cavity}

When $\varepsilon=0$, we obtain the equations that describe the ideal cavity without any pumping or losses. They are

$$
\dot{A}_{1}=-\mathrm{i} \kappa_{1} A_{1}+\mathrm{i} A_{2} A_{1}^{*}, \quad \dot{A}_{2}=\frac{\mathrm{i}}{2} A_{1}^{2},
$$

and can be derived via the formulas

$$
\dot{A}_{j}=-2 \mathrm{i} \frac{\partial H_{0}}{\partial A_{j}^{*}}, \quad j=1,2,
$$

from the Hamiltonian function

$$
H_{0}=\frac{1}{2} \kappa_{1}\left|A_{1}\right|^{2}-\frac{1}{4}\left(A_{1}^{2} A_{2}^{*}+A_{1}^{* 2} A_{2}\right) .
$$

An additional conserved quantity in (2) is the intensity of the light in the cavity,

$$
I=\frac{1}{4}\left|A_{1}\right|^{2}+\frac{1}{2}\left|A_{2}\right|^{2}
$$

The canonical transformation

$$
A_{1}= \pm \sqrt{2\left(2 I-|a|^{2}\right)} \mathrm{e}^{-\mathrm{i} \frac{\phi}{2}}, \quad A_{2}=a \mathrm{e}^{-\mathrm{i} \phi}
$$

reduces equations (2) into a planar Hamiltonian system for the complex variable $a$, and a quadrature for the angle $\phi$. From this reduced form, we calculate 
the family of heteroclinic solutions,

$$
\begin{aligned}
& A_{1}\left(t, I, \phi_{0}\right)= \pm \sqrt{2\left(2 I-\kappa_{1}^{2}\right)} \operatorname{sech}\left(\sqrt{2 I-\kappa_{1}^{2}} t\right) \mathrm{e}^{-\mathrm{i} \frac{\phi_{0}}{2}} \\
& A_{2}\left(t, I, \phi_{0}\right)=\left[\kappa_{1}+\mathrm{i} \sqrt{2 I-\kappa_{1}^{2}} \tanh \left(\sqrt{2 I-\kappa_{1}^{2}} t\right)\right] \mathrm{e}^{-\mathrm{i} \phi_{0}},
\end{aligned}
$$

which connect pairs of equilibria,

$$
A_{2}\left(-\infty, I, \phi_{0}\right)=\left(\kappa_{1}-\mathrm{i} \sqrt{2 I-\kappa_{1}^{2}}\right) \mathrm{e}^{-\mathrm{i} \phi_{0}}
$$

and

$$
A_{2}\left(\infty, I, \phi_{0}\right)=\left(\kappa_{1}+\mathrm{i} \sqrt{2 I-\kappa_{1}^{2}}\right) \mathrm{e}^{-\mathrm{i} \phi_{0}}
$$

that lie in the plane $A_{1}=0$ outside of the circle $2 I=\left|A_{2}\right|^{2}=\kappa_{1}^{2}$; see Figure 1. (The details of the calculation are similar to, but simpler than, those in [15].) By varying the parameter $I$ in the formulas (3), (4), and (5), we conclude that all the heteroclinic solutions (3) corresponding to a fixed value of the angle $\phi_{0}$ lie in a plane, and that their endpoints (4) and (5) all lie on the same straight line tangent to the circle $2 I=\left|A_{2}\right|^{2}=\kappa_{1}^{2}$. Furthermore, formulas (4), and (5) imply that the difference $\Delta(I)$ in the argument of the variable $A_{2}$ after each heteroclinic excursion (3) is equal to

$$
\Delta(I)=2 \arctan \frac{\sqrt{2 I-\kappa_{1}^{2}}}{\kappa_{1}} .
$$

\section{The pure second harmonic mode}

The pure second harmonic mode in the plane $A_{1}=0$ is invariant even for the full system (1). Its dynamics are governed by the equation

$$
\dot{A}_{2}=-\varepsilon\left(\mathrm{i} \kappa_{2}+\alpha\right) A_{2}+\varepsilon \gamma_{2}
$$

which is obtained from equations (1) in this plane. There is a single equilibrium in this plane at the point

$$
A_{1}=0, \quad A_{2}=\frac{\gamma_{2}}{\alpha+\mathrm{i} \kappa_{2}}
$$

which is, restricted to the $A_{1}=0$ plane, a spiral sink if $\alpha>0$ and $\kappa_{2} \neq 0$. Dynamics in this plane evolve on a slow, $\mathcal{O}(1 / \varepsilon)$ time scale. 


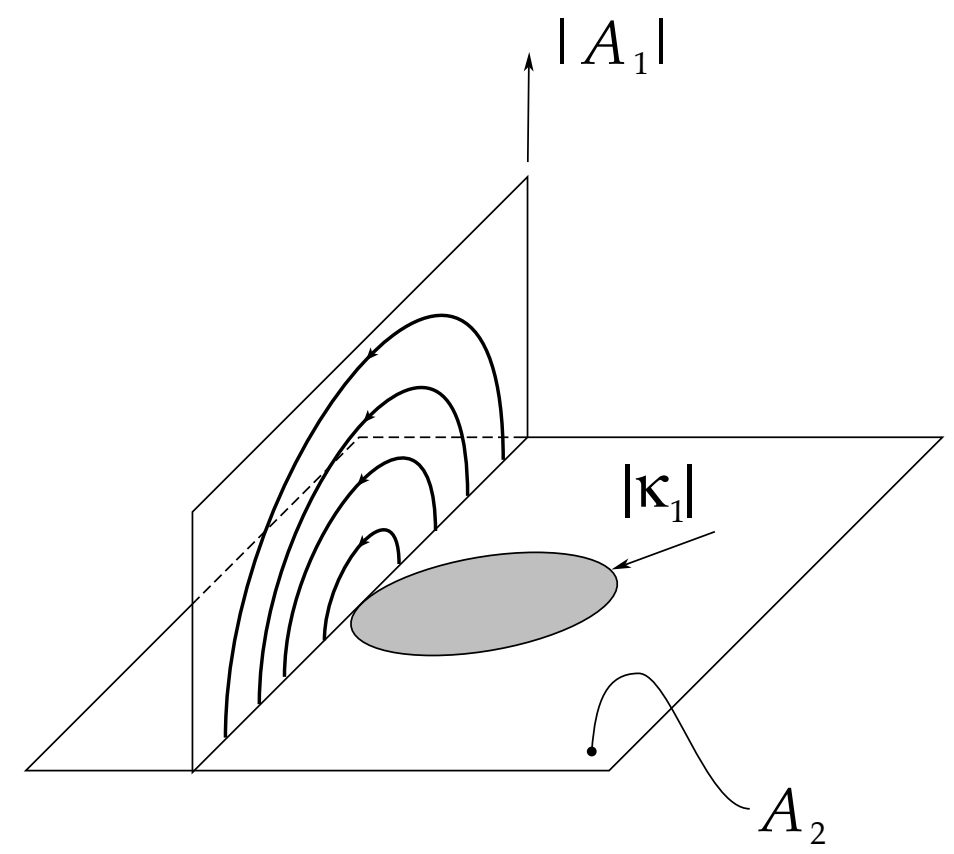

Fig. 1. Heteroclinic solutions (3) with a fixed value of the angle $\phi_{0}$.

\section{The $n$-pulse Melnikov function}

For system (1), a homoclinic orbit with $n$ pulses consists of two pieces. The first piece of such a homoclinic orbit is close to a string of $n$ consecutive heteroclinic orbits $\left(A_{1}, A_{2}\right)\left(t, \bar{I}, \bar{\phi}_{0}-j \Delta(\bar{I})\right)$, with $j=0, \ldots, n-1$. Here, the phase difference $\Delta(I)$ is given by equation (6). These heteroclinic orbits are called pulses. The string begins with the unstable manifold of the equilibrium point (8), that is, the heteroclinic orbit $\left(A_{1}, A_{2}\right)\left(t, \bar{I}, \bar{\phi}_{0}\right)$ given by formula $(3)$ with

$$
A_{2}\left(-\infty, \bar{I}, \bar{\phi}_{0}\right)=\left(\kappa_{1}-\mathrm{i} \sqrt{2 \bar{I}-\kappa_{1}^{2}}\right) e^{-i \bar{\phi}_{0}}=\frac{\gamma_{2}}{\alpha+\mathrm{i} \kappa_{2}} .
$$

This equation fixes the parameters $\bar{I}$ and $\bar{\phi}_{0}$ in all $n$ pulses of the string. The last heteroclinic orbit in the string lands back in plane $A_{1}=0$ at the point $A_{2}\left(\infty, \bar{I}, \bar{\phi}_{0}-(n-1) \Delta(\bar{I})\right)$. The second piece of a homoclinic orbit with $n$ pulses is close to the trajectory of the system (7) that connects the landing point $A_{2}\left(\infty, \bar{I}, \bar{\phi}_{0}-(n-1) \Delta(\bar{I})\right)$ of the last pulse back to the equilibrium (8).

A homoclinic orbit with $n$ pulses as described in the previous paragraph only exists if two further conditions are satisfied. First, its second piece, or rather the corresponding trajectory of the system $(7)$ that connects the point $A_{2}\left(\infty, \bar{I}, \bar{\phi}_{0}-(n-1) \Delta(\bar{I})\right)$ to the equilibrium (8), must avoid the circle $2 I=\left|A_{2}\right|^{2}=\kappa_{1}^{2}$ and its interior. This is because, otherwise, the mathematical arguments [6-8] used to establish the existence of such homoclinic orbits cease 
to be valid. Second, we must choose the parameters $\alpha, \gamma_{2}, \kappa_{1}$ and $\kappa_{2}$ so that the $n$-pulse Melnikov function $M_{n}\left(\bar{I}, \bar{\phi}_{0}\right)$, to be described next, vanishes along the string of heteroclinic orbits $\left(A_{1}, A_{2}\right)\left(t, \bar{I}, \bar{\phi}_{0}\right), \ldots,\left(A_{1}, A_{2}\right)\left(t, \bar{I}, \bar{\phi}_{0}-(n-\right.$ 1) $\Delta(\bar{I}))$.

The $n$-pulse Melnikov function [8] is the sum of the ordinary Melnikov functions calculated along each of the heteroclinic pulses (3). Here, the ordinary Melnikov function $[16,17]$ is

$$
M\left(I, \phi_{0}\right)=\int_{-\infty}^{\infty}\left(\vec{\nabla} H_{0} \cdot \vec{g}\right)\left(A_{1}\left(t, I, \phi_{0}\right), A_{2}\left(t, I, \phi_{0}\right)\right) \mathrm{d} t
$$

with

$$
\vec{\nabla}=\left(\frac{\partial}{\partial A_{1}}, \frac{\partial}{\partial A_{1}^{*}}, \frac{\partial}{\partial A_{2}}, \frac{\partial}{\partial A_{2}^{*}}\right),
$$

and $\vec{g}$ the $\mathcal{O}(\varepsilon)$ part of the vector field (1). (See also the exposition in [18], and the references to original works cited there.) An easy calculation, similar to that in [15], shows that for our model

$$
M\left(I, \phi_{0}\right)=\gamma_{2} \operatorname{Im}\left[A_{2}\left(-\infty, I, \phi_{0}\right)-A_{2}\left(\infty, I, \phi_{0}\right)\right]+2 \alpha \kappa_{1} \sqrt{2 I-\kappa_{1}^{2}},
$$

where Im denotes the imaginary part of a complex number, so that the $n$-pulse Melnikov function is given by the expression

$$
\begin{gathered}
M_{n}\left(I, \phi_{0}\right)=\gamma_{2} \operatorname{Im}\left[A_{2}(-\infty,\right. \\
\left.\left., I, \phi_{0}\right)-A_{2}\left(\infty, I, \phi_{0}-(n-1) \Delta(I)\right)\right] \\
+2 n \alpha \kappa_{1} \sqrt{2 I-\kappa_{1}^{2}} .
\end{gathered}
$$

\section{Homoclinic orbits with $n$-pulses in the shape of a regular $n$-Gon}

We consider homoclinic orbits whose shape is nearly that of a regular $n$-gon or a regular star with $n$ vertices. By equations (4) and (5), adjacent vertices of such an $n$-gon or star lie on straight lines tangent to the circle $\left|A_{2}\right|^{2}=\kappa_{1}^{2}$. Therefore, $I=\bar{I}$ must be chosen so that the angle difference $\Delta(\bar{I})$ from equation (6) satisfies the equation

$$
\Delta(\bar{I})=2 \arctan \frac{\sqrt{2 \bar{I}-\kappa_{1}}}{\kappa_{1}}=\operatorname{sign}\left(\kappa_{1}\right) \frac{2 \pi m}{n}+2 \delta,
$$

where $n>2$ and $m$ are relatively prime integers with $m<n / 2$, and $\delta$ is a small number. 


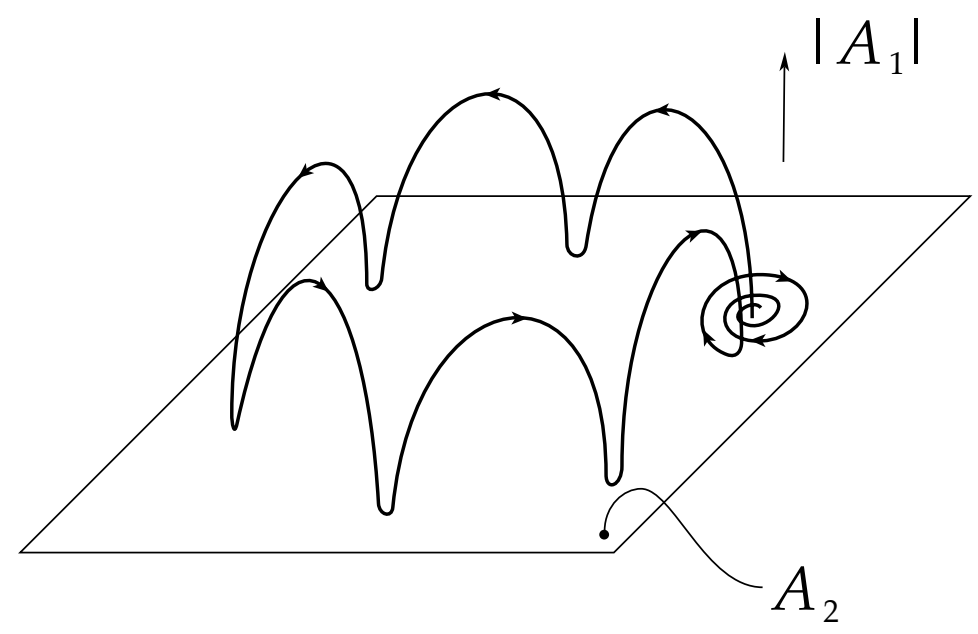

Fig. 2. A homoclinic orbit in the shape of a hexagon.

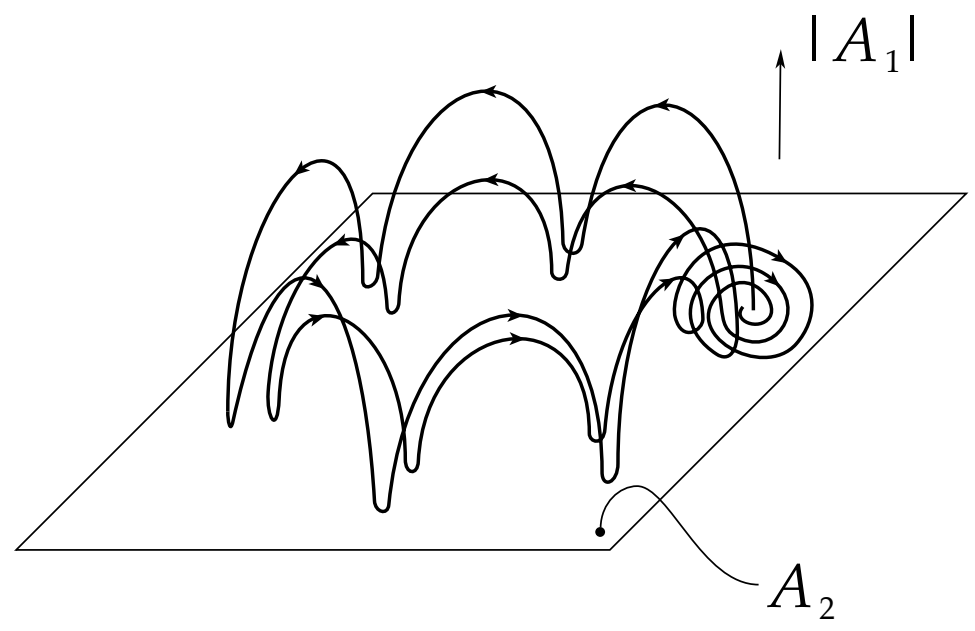

Fig. 3. A two-bump homoclinic orbit in the shape of a hexagon.

Equations (9), (10), and (11) imply that if we choose the parameters $\kappa_{1}$, $\kappa_{2}$, and $\delta$, we can compute $\alpha$ and $\gamma_{2}$ so that there exists an $n$-pulse, Silnikov type orbit [9-13] homoclinic to the equilibrium point (8), whose sequence of pulses encircles the origin $m$ times before easing into a spiral of size $2 \delta \bar{I}$, which lies close to the plane $A_{1}=0$, and slowly winds back into the equilibrium (8); see Figure 2. Specifically, $\alpha$ and $\gamma_{2}$ are given by the expressions

$$
\alpha=\operatorname{sign}\left(\kappa_{1}\right) \frac{2 n \kappa_{2}}{\sin \frac{2 \pi m}{n}} \delta^{2}+\mathcal{O}\left(\delta^{3}\right), \quad \gamma_{2}= \pm \frac{\kappa_{1} \kappa_{2}}{\cos \frac{2 \pi m}{n}}+\mathcal{O}(\delta)
$$

The first of these equations shows that we must choose the detunings $\kappa_{1}$ and $\kappa_{2}$ to have the same sign in order for these homoclinic orbits to exist in the physical regime $\alpha>0$. 
Besides the just-described $n$-pulse homoclinic orbits in which all pulses follow one another in one group or bump, we can also find homoclinic orbits of a similar shape in which several bumps, each consisting of $n$-consecutive pulses, follow one another, being interspersed with slow, spiral-like segments that lie close to the plane $A_{1}=0$. The only restriction on these slow segments is that no part of any of them may lie inside the circle $2 I=\left|A_{2}\right|^{2}=$ $\left|\kappa_{1}\right|^{2}$. We can form a $k$-bump homoclinic orbit, with each bump containing $n$ pulses, as follows [7]. Its first bump emanates from the equilibrium (8). All its other bumps arise from essentially the same zero of the $n$-pulse Melnikov function as the first bump. In particular, they take off from the plane $A_{1}=0$ near a curve $A_{2}\left(-\infty, I, \phi_{0}(I)\right)$, whose existence easily follows from the simple zero of the Melnikov function at $I=\bar{I}$ and $\phi_{0}=\bar{\phi}_{0}$, so that $\phi_{0}(\bar{I})=\bar{\phi}_{0}$. Every $k$-bump homoclinic orbit can be assigned a symbol sequence, $\sigma_{1} N_{1} \tau_{2} \sigma_{2} N_{2} \ldots \tau_{k-1} \sigma_{k-1} N_{k-1} \tau_{k} \sigma_{k}$, in which $\sigma_{j}= \pm$ denotes the sign of the real part of the $A_{1}$ coordinate of the $j$-th bump, $\tau_{j}= \pm$ indicates whether the value of $I$ at the takeoff of the $j$-th bump is $I>\bar{I}$ or $I<\bar{I}$, and $N_{j}$ denotes the number of times the slow segment that connects the $j$-th and $(j+1)$-st pulse winds around the point (8). Each such homoclinic orbit is of Silnikov type; see Figure 3.

\section{Multi-bump homoclinic orbits with an even number of pulses in each bump}

We now consider the limit in which the ratio between the detuning $\kappa_{1}$ and the distance $\sqrt{2 \bar{I}}$ of the equilibrium point (8) from the origin is very small. In this case, the corresponding phase difference $\Delta(I)$ from equation (6) is close to $\pi$ for $I$ close to $I=\bar{I}$. We define $\nu(I)=\kappa_{1} / \sqrt{2 I}$, and $\bar{\nu}=$ $\nu(\bar{I})$, and consider the case $\nu(I), \bar{\nu} \ll 1$. Re-computing the $2 m$-pulse Melnikov function $M_{2 m}\left(\bar{I}, \bar{\phi}_{0}\right)$ along a string of $2 m$ consecutive pulses emerging from the equilibrium (8), Taylor-expanding in the small parameter $\bar{\nu}$, and setting $M_{2 m}\left(\bar{I}, \bar{\phi}_{0}\right)=0$ now yields the formula

$$
\alpha=m \kappa_{2} \bar{\nu}+\mathcal{O}\left(\bar{\nu}^{2}\right) .
$$

For this particular value of $\alpha$, a Šilnikov-type homoclinic orbit [9-13] with $2 m$ pulses connects the equilibrium point (8) to itself. The pulses of this homoclinic orbit jump between an $\mathcal{O}(\bar{\nu})$-sized neighborhood of the point $(8)$ and an $\mathcal{O}(\bar{\nu})$ sized neighborhood of its antipodal point, until this orbit finally spirals into the point (8). Note that we must again have $\kappa_{2} \bar{\nu}>0$, that is $\kappa_{1} \kappa_{2}>0$, in order that $\alpha>0$, which is the physical regime.

We now compute the curves along which $2 n$-pulse strings of unperturbed heteroclinic orbits take off from and land on the $A_{2}$-plane for general $n$. We 
will use these strings as building blocks in constructing orbits homoclinic to the point (8) with many bumps, each bump consisting of an even, possibly different, number of pulses. In particular, again re-computing the $2 n$-pulse Melnikov function $M_{2 n}\left(I, \phi_{0}\right)$, Taylor-expanding in $\nu(I)$, setting $M_{2 n}\left(I, \phi_{0}\right)=$ 0 , assuming $\nu(I)=\bar{\nu}+\mathcal{O}\left(\bar{\nu}^{2}\right)$, and using formula (12), we compute that the takeoff and landing curves in the plane $A_{1}=0$ for the persisting $2 n$-pulse strings of heteroclinic orbits (3) lie at

$$
\begin{aligned}
& A_{2}\left(-\infty, I, \phi_{0}(I)\right)=-\operatorname{sign}\left(\gamma_{2} \kappa_{2}\right) {[\mathrm{i} \sqrt{2 I}} \\
&+\sqrt{2 \bar{I}}(m-2 n) \bar{\nu}]+\mathcal{O}\left(\bar{\nu}^{2}\right),
\end{aligned}
$$

and

$$
\begin{aligned}
A_{2}\left(\infty, I, \phi_{0}(I)-(n-1) \Delta(I)\right)=- & \operatorname{sign}\left(\gamma_{2} \kappa_{2}\right)[\mathrm{i} \sqrt{2 I} \\
& +\sqrt{2 \bar{I}}(m+2 n) \bar{\nu}]+\mathcal{O}\left(\bar{\nu}^{2}\right) .
\end{aligned}
$$

In the rest of this discussion, we only consider homoclinic orbits in the case when $\gamma_{2} \kappa_{2}<0$, because the case when $\gamma_{2} \kappa_{2}>0$ yields almost identical results. We construct a homoclinic orbit that connects the equilibrium (8) at $A_{2}=A_{2}\left(-\infty, \bar{I}, \bar{\phi}_{0}\right)=\sqrt{2 \bar{I}}(\mathrm{i}-m \bar{\nu})+\mathcal{O}\left(\bar{\nu}^{2}\right)$ to itself as follows. The first bump of this orbit stays near a string of $2 m$ pulses emanating from this equilibrium, and returns to the $A_{2}$-plane near the point $A_{2}=\sqrt{2 \bar{I}}(\mathrm{i}+3 m \bar{\nu})+\mathcal{O}\left(\bar{\nu}^{2}\right)$. After that, the homoclinic orbit continues near this plane along a tight spiral that stays $\mathcal{O}\left(\bar{\nu}^{2}\right)$ close to the circle $\left|A_{2}-\sqrt{2 \bar{I}}(\mathrm{i}-m \bar{\nu})\right|=4 m \bar{\nu}$ for $N_{1}$ revolutions around the equilibrium point (8), and then takes off again along the first pulse of the second bump. For general $j$, the $j$-th bump takes off near the intersection point of the circle $\left|A_{2}-\sqrt{2 \bar{I}}(\mathrm{i}-m \bar{\nu})\right|=R_{j-1} \bar{\nu}$ and the line (13), where $n=n_{j}$ is the number of its pulses, and lands near the intersection point of the circle $\left|A_{2}-\sqrt{2 \bar{I}}(\mathrm{i}+3 m \bar{\nu})\right|=R_{j-1} \bar{\nu}$ and the line (14) with $n=n_{j}$. The piece of the spiral that follows this bump lies $\mathcal{O}\left(\bar{\nu}^{2}\right)$ close to the circle $\left|A_{2}-\sqrt{2 \bar{I}}(\mathrm{i}-m \bar{\nu})\right|=R_{j} \bar{\nu}$, where $R_{j}=4 \sqrt{m\left(m+\sum_{l=2}^{j} n_{l}\right)}$, and makes $N_{j}$ revolutions around the origin. Moreover, we must have

$$
n_{j}<m+\frac{R_{j-1}}{2}=m+2 \sqrt{m\left(m+\sum_{l=2}^{j-1} n_{l}\right)} .
$$

If, as in the previous section, we let $\sigma_{j}= \pm$ denote the sign of the real part of the $A_{1}$ coordinate of the $j$-th bump, and $\tau_{j}= \pm$ indicate whether the value of $I$ at the takeoff of the $j$-th bump is $I>\bar{I}$ or $I<\bar{I}$, we can conclude that we can form a homoclinic orbit connecting the equilibrium point (8) to itself in the way just described for every sequence of symbols and integers $\sigma_{1} m N_{1} \tau_{2} \sigma_{2} n_{2} N_{2} \ldots \tau_{k-1} \sigma_{k-1} n_{k-1} N_{k-1} \tau_{k} \sigma_{k} n_{k}$; see Figure 4 . 


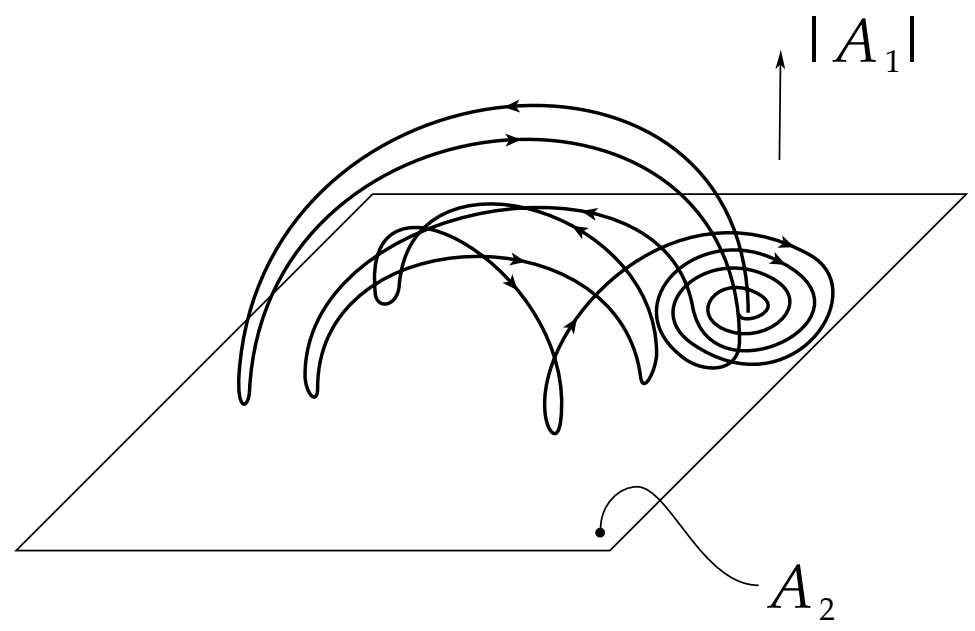

Fig. 4. A homoclinic orbit with two bumps, the first containing two pulses and the second four.

\section{Conclusion}

We have exhibited two large families of Šilnikov-type homoclinic orbits [913] that are present in the model (1). Even though we have assumed that only the second-harmonic mode in the cavity is pumped in this model, it is easy to see that the calculations and results would be nearly identical if the fundamental mode was also pumped by a term of the form $\varepsilon \gamma_{1}$. We should also remark that all the homoclinic orbits discussed in the previous two sections exist in the limit of small $\varepsilon$. Thus, we expect that for any finite value of $\varepsilon$, we should only be able to obtain a finite number of such homoclinic orbits. However, this number should increase with decreasing $\varepsilon$.

Well-known arguments [12] show that the homoclinic orbits that we have found imply the presence of a Smale horseshoe return map [14], and thus chaotic dynamics. The orbits generated by the Smale horseshoe map are all unstable, and thus only exhibit transient chaos. However, the presence of homoclinic orbits in the phase space of the model (1) is also one likely mechanism responsible for the occurrence of a strange attractor seen numerically at $\mathcal{O}(1)$ values of $\varepsilon$. In particular, a period-doubling route to such an attractor was computed in [4], and it is not inconceivable that a Šilnikov mechanism could be responsible for this route. (In this respect, see the exposition and references to the orginal works in [18].) The strange attractor has also been observed experimentally [19]. A numerical investigation of a possible connection between the homoclinic orbits described in this letter and the strange attractor, in particular, a continuation of these homoclinic orbits to $\mathcal{O}(1)$ values of $\varepsilon$ where this atrractor exists, is in progress and will be presented elsewhere. 


\section{Acknowledgements}

A. A. acknowledges support from the U.S. Department of Energy through grant DE-FG02-93ER25163, and the U.S. Air Force Office of Scientific Research through grant F4962094-1-0007. D. D. H. acknowledges support by the U.S. Department of Energy through its Office of Basic Energy Science program in Applied Mathematical Sciences under contract KC-07-01-01, and the Los Alamos National Laboratory under contract W-7405-ENG-36. G. K. and I. T. thank the Theoretical Division and Center for Nonlinear Studies at the Los Alamos National Laboratory for their hospitality and support during the summers of 1992 through 1996, and acknowledge support from the U.S. Department of Energy through grant DE-FG02-93ER25154, the National Science Foundation through grants DMS-9403750, DMS-9502142, and DMS-9510728, and the Alfred P. Sloan Foundation through a Sloan Research Fellowship.

\section{References}

[1] P. D. Drummond, K. J. McNeill, and D. F. Walls, Opt. Acta, 27 (1980), 321.

[2] P. Mandel and X. G. Wu, J. Opt. Soc. Am. B, 3 (1986), 940.

[3] V. Zehnle and P. Mandel, Opt. Comm., 66 (1988), 216.

[4] L. A. Lugiato, C. Oldano, C. Fabre, E. Giacobino, and R. J. Horowicz, Il Nuovo Cimento D, 10 (1988), 959.

[5] S. Trillo and G. Assanto, Opt. Lett., 19 (1994), 1825.

[6] G. Kovačič, SIAM J. Math. Anal., 26 (1995), 1611.

[7] T. J. Kaper and G. Kovačič, Trans. AMS, 348 (1996), 3835.

[8] R. Camassa, G. Kovačič, and S.-K. Tin, A Melnikov method for homoclinic orbits with many pulses, to appear in Arch. Rat. Mech. Anal., (1996).

[9] L. P. Šilnikov, Sov. Math. Dokl., 6 (1965), 163.

[10] L. P. Šilnikov, Sov. Math. Dokl., 8 (1967), 102.

[11] L. P. Šilnikov, Math. USSR Sb. 10 (1970), 91.

[12] C. Tresser, Ann. Inst. Henri Poincaré, 40 (1984), 440.

[13] B. Deng, J. Diff. Eqns., 102 (1993), 305.

[14] S. Smale, in Differential and Combinatorial Topology, S. S. Cairns (Ed.), 63-80, Princeton Univ. Press: Princeton, (1963).

[15] G. Kovačič and T. A. Wettergren, ZAMP, 47 (1996), 221. 
[16] H. Poincaré, Les Méthodes Nouvelles de la Mécanique Celeste, 3 vols., GauthierVillars: Paris, (1899).

[17] V. K. Melnikov, Trans. Moscow Math., 12 (1963), 1.

[18] S. Wiggins, Global Bifurcations and Chaos: Analytical methods, Springer-Verlag: New York, (1988).

[19] C. M. Savage and D. F. Walls, Opt. Acta, 30 (1983), 557. 